\title{
Rolf Wynn: The Linguistics of Doctor-Patient Communication. Oslo: Novus Press, 1995
}

Most of us who have been to the doctor lately probably agree that research in doctor-patient communication is not only a valid but also a much-needed discipline. Doctor-patient communication is difficult - just look at the way consultations tend to produce more unanswered than answered questions no matter how prepared the patient is for the session.

The author of the book, Rolf Wynn, gives the following reason why research into doctor-patient communication should be pursued:

"Doctors are concerned with making a correct diagnosis and pursuing the most efficient treatment, processes invariably connected to the doctor-patient interaction. Doctor-patient communication is especially important in those numerous cases where there are few if any 'objective' signs or laboratory results, and where the diagnosis and treatment have to be based solely on the information acquired from doctor-patient communication'. (p. 9)

However, Wynn's book (which is a slightly revised version of his Master's thesis in English from 1993) is not dealing with doctor-patient communication research itself but rather the methodology of doctor-patient communication research/analysis.

Thus the aim of the book is to compare and contrast two of the main approaches to doctor-patient communication research, namely a sociolinguistic approach (ethnomethodological conversation analysis (ECA)) and a medical approach (Bales' Interaction Process Analysis (BIPA) and Byrne \& Long)

According to Wynn such a comparison is interesting because few doctors, using the medical approach for analysing doctor-patient interaction, display any knowledge of linguistics and the sociolinguistic approach. And at the same time few linguists seem to display thorough knowledge of modern medical practices and the medical approach to doctor-patient interaction analysis. Being a student of both medicine and linguistics, Wynn acts as the mediator and presents the reader with the wide range of the tools available within the medical and the sociolinguistic approaches to doctor-patient communication research.

The book consists of 8 chapters. Chapter 1 introduces the aim of the book (mentioned above), presents the terminology, definitions and abbreviations used and accounts for the organisation of the book.

Chapter 2 introduces the two main approaches to doctor-patient communication, the medical approach (BIPA and Byrne \& Long) on the one hand and the sociolinguistic approach (ECA) on the other. The chapter provides an excellent, but brief, overview of the two approaches such as their main characteristics and results obtained in connection with the analyses of doctor-patient 
communication. However, when it comes to a critical view of the methodological problems of the two approaches, the author seems somewhat biased. A large number of the weaknesses of the medical approach are listed whereas the weaknesses - which no doubt the sociolinguistic approach also possesses - are not commented on in this chapter.

According to Wynn there are two major themes which people doing doctorpatient communication research tend to focus on - namely power distribution and the relationship between 'linguistic' variables and the outcome of the interaction/medical treatment. Chapter 3 deals with these themes - not only because of their centrality but also because researchers within the sociolinguistic approach tend to focus on power distribution in interaction and researchers within the medical approach tend to focus on the outcome of the interaction. Thus chapter 3 not only elaborates on central themes in connection with doctor-patient communication research but does so while keeping the purpose of the book in mind - namely that of contrasting and comparing the two methods of analysis. However, again we see the same tendency as in chapter 2 of presenting the analyses of power distribution in a favourable light (the sociolinguistic approach) whereas the out-come based analyses of doctor-patient interaction are heavily criticised (the medical approach). For example when considering the out-come based analyses, Wynn addresses the difficulty of correlating the out-come of medical treatments (e.g. satisfaction or cure) to linguistic variables and he therefore questions the possibility of doing outcome based analyses. However, in connection with the analyses of power distribution Wynn does not even consider whether power distribution is in fact such a major hindrance to communication, as the sociolinguistic approach seems to suggest. In other words he does not discuss whether there are other and perhaps more relevant reasons behind miscommunication such as time pressure, the use of medical terminology, the mental state of the patient, personal characteristics - both of doctor and patient, etc. Furthermore, he does not comment on the difficulty of correlating power distribution and language use which is not unproblematic either.

Chapter 4 presents different methods for obtaining data for analysis of doctor-patient communication (questionnaires, interviews, one-way mirrors, and audio and video recordings) and comments on the advantages and disadvantages of the various methods. It also presents a brief introduction to some of the problems in connection with data collection such as code-switching, the setting of data collection, the sampling of doctors, patients and recordings and finally the process of transcription. The chapter relates the different methods for data collection to the two systems of analysis and provides the reader with a clear idea of the way the sociolinguistic and the medical approaches deal with the question of data. At the same time the chapter appears as a useful 
guide on how to collect data - in the sense that the potential researcher gets a clear idea of the possibilities and limitations choices in data collection may have.

After data collection the researcher is faced with yet another problem namely that of coding the meaning of the utterances recorded. Chapter 5 discusses aspects of coding and introduces two approaches for decoding utterances; the etic approach (often used by researchers within the medical tradition) and the emic approach (often used by researchers within the sociolinguistic tradition). The etic approach uses the linguistic features within an utterance for interpreting the meaning (e.g. Verb+Subject order indicates that it is a question) whereas the emic approach relies on the context of the utterance. Wynn finds the etic approach too narrow but points also to some of the weaknesses of an emic approach and says that "...finding the probable intent of an interactant is very difficult, if possible at all” (p. 77). A statement which, I am sure, would make most medical researchers even more loyal to their etic approach.

In spite of the difficulties associated with coding utterances, researchers still try to code parts of doctor-patient interaction. In chapter 6 Wynn looks at a few linguistic variables of doctor-patient interaction (questions, interruptions and turn-time) which the sociolinguistic approach tends to favour in its doctorpatient research. Chapter 6 accounts for the way the sociolinguistic approach relates power distribution to linguistic features (such as questions, interruptions, etc.). However, in doing so Wynn also indirectly reveals the weaknesses of such an alleged relationship as it appears from the examples discussed that a particular linguistic phenomenon cannot and should not always be interpreted as a sign of more or less power.

In chapter 7 Wynn deals with two approaches to the analysis of data - the qualitative and the quantitative approaches and evaluates their strengths and weaknesses. In doing so he once again contrasts the medical approach to doctor-patient communication with the sociolinguistic approach because there is a tendency in the medical tradition (e.g. BIPA) of using quantitative approaches and a tendency in the sociolinguistic tradition (ECA) of using qualitative approaches. The chapter provides a good overview of the weaknesses and strengths of the two approaches to the analysis of data which people embarking on doctor-patient communication research for the first time definitely can benefit from.

Chapter 8 summarises main results and draws conclusions. First of all Wynn comments on the differences between the two approaches to doctor-patient communication described throughout the preceding 7 chapters. However, more importantly he uses the conclusion for recommending a combination of the two approaches to doctor patient-communication. "The possibility of mix- 
ing these aspects of ECA [turn-taking, adjacency pairs, questions, etc) with aspects of the quantitative approaches is interesting and refreshing" (p. 115). This leads me to suggest that maybe the aim of this book is not simply as stated in the introduction to "compare and contrast different, alternative ways of doing doctor-patient interaction analysis" (p. 10), but rather to introduce two research traditions to each other and make them aware of the possibility of joining forces in the area of doctor-patient communication. Or - due to Wynn's tendency of emphasising weaknesses of the medical approach and strengths of the sociolinguistic approach - to convince researchers within the medical tradition of the advantages of the sociolinguistic approach. Whether Wynn's mission is successful is not for me to decide. However, being both a member of a linguistic as well as a medical community, Wynn stands a good chance of reaching both linguists and doctors.

Nevertheless, there are other people besides those already working with doctor-patient communication research who may benefit from this book. The book provides an excellent introduction to methodological aspects such as data collection, coding, analysis etc. And I would, therefore, strongly recommend the book to Ph.D.-students not only within the field of doctor patient communication (to whom the book of course would be extremely relevant) but also within any field of research where theoretical and methodological considerations are a must. 